\title{
Chaotic Synchronization of Nonlinear-Coupled Fractional-order Liu System and Secure Communication
}

\author{
Birong Xu \\ School of Mechanical and Electrical Engineering, Wuyi University, \\ Wuyishan 354300, China \\ xubirong1@163.com
}

\begin{abstract}
The synchronization of nonlinear-coupled fractional-order Liu systems is verified theoretically and numerically when the sum of the coupling coefficients of systems is $\alpha+\beta=1$. Numerical simulations coincide with the theoretical analysis, and show fractional order and linear matrix influence chaotic synchronization. Furthermore, this method is applied in secure communication, and a chaotic masking system is designed.

Keywords: Nonlinear-coupled Method, Fractional-order Liu Chaotic System, Chaotic Synchronization, Secure Communication

\section{Introduction}

Fractional-order chaotic systems have become a hot topic recently. Some fractional-order differential systems, such as Duffing system ${ }^{[1]}$, Liu system ${ }^{[2]}$, Newton-Leipnik system ${ }^{[3]}$ and so on, have been found. Their chaotic behaviors were presented. Sensitivity to initial conditions is an important characteristic of chaotic systems. The chaotic systems are usually difficult to be controlled or synchronized. The synchronization of the fractional-order chaotic systems has been considered as an important and challenging problem, and become more and more interesting for researchers ${ }^{[4,5]}$. Therefore, the synchronization of the fractional order Liu system is realized by the nonlinear-coupled synchronization method in this paper. Moreover, the fractional order and linear Matrix influence the synchronization will be investigated. Then, the secure communication scheme is proposed based on this method.
\end{abstract}

\section{Nonlinear-coupled synchronization method for the fractional-order chaotic system}

Let us consider the fractional-order chaotic system 


$$
\frac{d^{q} X}{d t^{q}}=F(X(t), t)
$$

where $0<q \leq 1$ is the fractional-order time derivatives, $X(t) \in R^{n}$ is the state vector and $F: R^{n} \rightarrow R^{n}$ is a continuous vector function. $F(X(t), t)$ can be written as

$$
F(X(t), t)=K X(t)+N(X(t), t)
$$

where $N(X(t), t)$ is the nonlinear term of $F(X(t), t)$ and $K$ is constant matrix, which has negative real part eigenvalues.So, the system (1) can be described as

$$
\frac{d^{q} X}{d t^{q}}=K X(t)+N(X(t), t) .
$$

There are two fractional-order chaotic systems with same structure as follow:

$$
\begin{aligned}
& \frac{d^{q} X_{1}}{d t^{q}}=K X_{1}(t)+N\left(X_{1}(t), t\right), \\
& \frac{d^{q} X_{2}}{d t^{q}}=K X_{2}(t)+N\left(X_{2}(t), t\right) .
\end{aligned}
$$

With nonlinear-coupled synchronization method ${ }^{[6]}$, we can construe two systems as follow:

$$
\begin{aligned}
& \frac{d^{q} X_{1}}{d t^{q}}=K X_{1}(t)+N\left(X_{1}(t), t\right)+\alpha\left[N\left(\left(X_{2}(t), t\right)-N\left(X_{1}(t), t\right)\right],\right. \\
& \frac{d^{q} X_{2}}{d t^{q}}=K X_{2}(t)+N\left(X_{2}(t), t\right)+\beta\left[N\left(\left(X_{1}(t), t\right)-N\left(X_{2}(t), t\right)\right],\right.
\end{aligned}
$$

where $\alpha$ and $\beta$ are the coupling coefficients of system.

Theorem: Given fractional-order systems (6) and (7), their synchronization can be achieved if the sum of the coupling coefficients of system is $\alpha+\beta=1$.

Proof: Let the synchronization error between systems (6) and (7) be

$$
e=X_{1}-X_{2} \text {. }
$$

Hence, the dynamic equation of the synchronization error can be obtained easily from Eq. (6) and (7), and it is expressed as follow: 


$$
\frac{d^{q} e}{d t^{q}}=K e+(\alpha+\beta-1)\left[N\left(\left(X_{2}(t), t\right)-N\left(X_{1}(t), t\right)\right]\right.
$$

Because $\alpha+\beta=1$, Eq.(9) is written as

$$
\frac{d^{q} e}{d t^{q}}=K e
$$

Since $K$ has negative real part eigenvalues, which is $\left|\arg \lambda_{i}\right|>\frac{\pi}{2}$. It is $0<q \leq 1$, so there is $\left|\arg \lambda_{i}\right|>\frac{q \pi}{2}$. According to Matignon's theorem, the equilibrium point $e_{i}=0 \quad(i=1,2, \cdots, n)$ of the error dynamics(9) is asymptotically stable, which implies that the synchronization between systems(6) and (7) can be achieved.

\section{Numerical simulation of the fractional-order Liu system}

We take the fractional-order Liu system for example to show the effectiveness of the proposed method. The fractional-order Liu system[2] is described as

$$
\left\{\begin{array}{l}
\frac{d^{q} x_{1}}{d t^{q}}=10\left(x_{2}-x_{1}\right), \\
\frac{d^{q} x_{2}}{d t^{q}}=40 x_{1}-x_{1} x_{3}, \\
\frac{d^{q} x_{3}}{d t^{q}}=4 x_{1}^{2}-2.5 x_{3} .
\end{array}\right.
$$

Eq. (11) can be written as Eq. (3), in which

$$
K=\left[\begin{array}{ccc}
-10 & 10 & 0 \\
-5 & 0 & 0 \\
0 & 0 & -2.5
\end{array}\right], N=\left[\begin{array}{c}
0 \\
45 x_{1}-x_{1} x_{3} \\
4 x_{1}^{2}
\end{array}\right]
$$

Hence, via the scheme in the paper, we give the coupled systems as following: 


$$
\begin{aligned}
& {\left[\begin{array}{l}
\frac{d^{q} x_{1}}{d t^{q}} \\
\frac{d^{q} x_{2}}{d t^{q}} \\
\frac{d^{q} x_{3}}{d t^{q}}
\end{array}\right]=\left[\begin{array}{c}
10\left(x_{2}-x_{1}\right) \\
40 x_{1}-x_{1} x_{3} \\
4 x_{1}^{2}-2.5 x_{3}
\end{array}\right]+\alpha\left[\begin{array}{c}
0 \\
\left(45 y_{1}-y_{1} y_{3}\right)-\left(45 x_{1}-x_{1} x_{3}\right) \\
4 y_{1}^{2}-4 x_{1}^{2}
\end{array}\right],} \\
& {\left[\begin{array}{c}
\frac{d^{q} y_{1}}{d t^{q}} \\
\frac{d^{q} y_{2}}{d t^{q}} \\
\frac{d^{q} y_{3}}{d t^{q}}
\end{array}\right]=\left[\begin{array}{c}
10\left(y_{2}-y_{1}\right) \\
40 y_{1}-y_{1} y_{3} \\
4 y_{1}^{2}-2.5 y_{3}
\end{array}\right]+\beta\left[\begin{array}{c}
\left(45 x_{1}-x_{1} x_{3}\right)-\left(45 y_{1}-y_{1} y_{3}\right) \\
4 x_{1}^{2}-4 y_{1}^{2}
\end{array}\right] .}
\end{aligned}
$$

The eigenvalues of $K$ are -2.5 , and $-5 \pm 5 i$. There are $|\arg (-2.5)|=\pi>\frac{q \pi}{2}$ and $|\arg (-5 \pm 5 i)|=\frac{3 \pi}{4}>\frac{q \pi}{2}$. According to the above theorem, the systems (12) and (13) can realize synchronization if the sum of the coupling coefficients of systems is $\alpha+\beta=1$. When the initial conditions are $\left(x_{10}, x_{20}, x_{30}\right)=(2,1,3)$ and $\left(y_{10}, y_{20}, y_{30}\right)=(15,6.5,7)$, and the fractional-order is $\mathrm{q}=0.9$, then the coupling coefficients of system are $\alpha=0.3$ and $\beta=0.7$, the chaotic attractors of the couple systems is shown in Fig. 1(a). System (12) and system (13) have the same chaotic behavior. The corresponding error curve is displayed in Fig. 1(b), which indicates that the synchronization of systems (12) and (13) realizes.

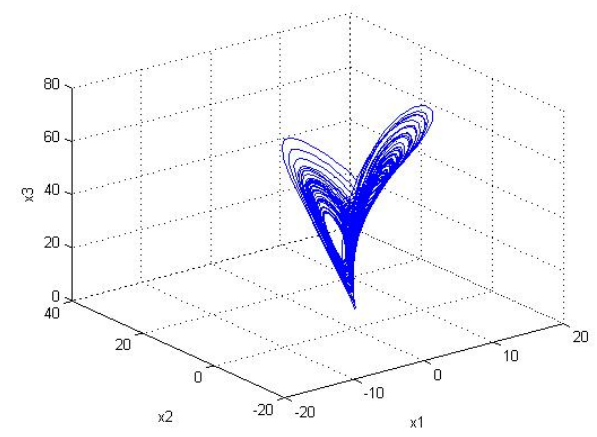

(a) 


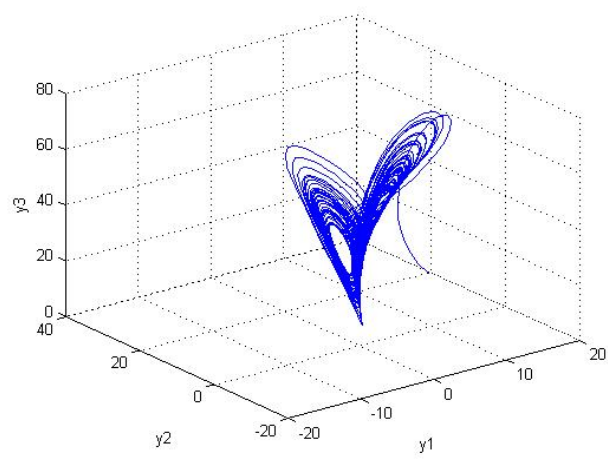

(b)

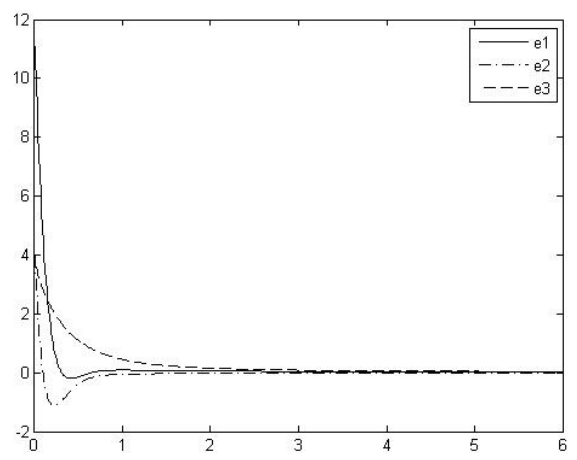

(c)

Fig. 1. Synchronized curves of the coupled systems (12) and (13) with q=0.9. (a) Chaotic attractors of system (12); (b) Chaotic attractors of system (13);(c) Synchronized errors of the coupled systems

Next, we will further investigate the impact of the fractional order and linear Matrix on chaotic synchronization for the coupled Liu systems. Fig. 2 shows the synchronization results when the orders are $q=0.9,0.95$ and 0.99 . The larger the order is, the faster the synchronization achieves. Then the linear Matrix is different, and the synchronization result is different too. Let $a$ to represent the element in the second row and the first column of the linear matrix. As is described in table $1, a$ is taken as $-1,-2$, and -5 , the synchronization results are shown in Fig. 3. With $a$ reducing, the systems cost less time to attain synchronization, and all the synchronizations can be eventually achieved at different linear matrix, which has negative real part eigenvalues. 




(a)

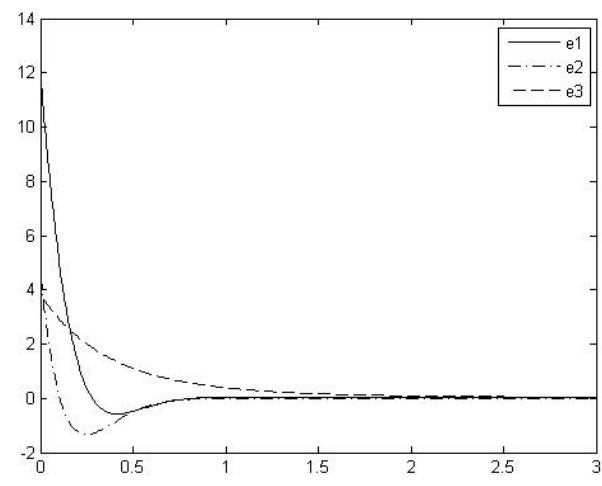

(b)

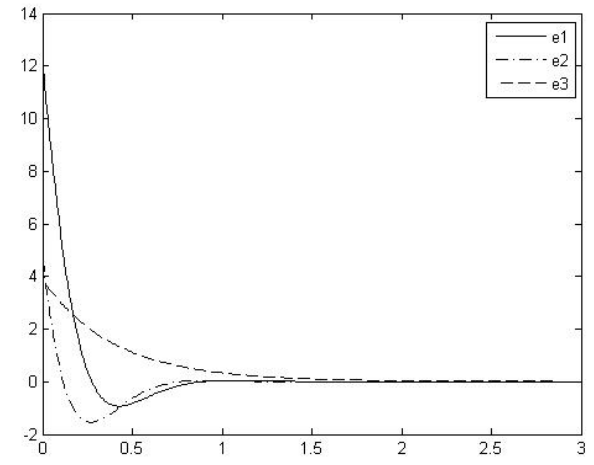

(c)

Fig. 2. Synchronized errors of the coupled systems (12) and (13) with different orders. (a) q=0.9; (b) q=0.95; (c) q=0.99. 




(a)

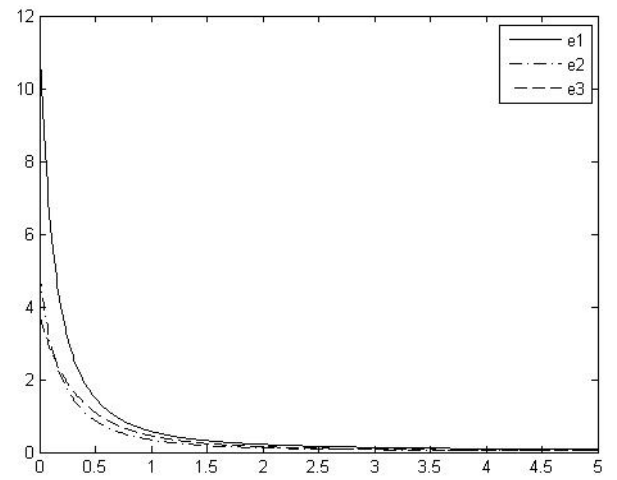

(b)

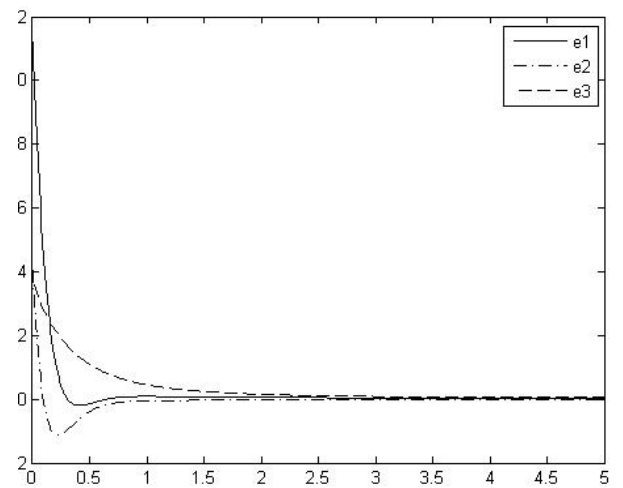

(c)

Fig. 3. Synchronized errors of the coupled systems (12) and (13) with different matrixes. (a) $a=-1$; (b) $a=-2$; (c) $a=-5$. 


\section{The secure communication}

In this section, we will display a secure communication scheme based on above method. By the chaotic making technique, the drive system of the communication scheme is the same as system 12 , and the response system is as following:

$$
\left[\begin{array}{c}
\frac{d^{q} y_{1}}{d t^{q}} \\
\frac{d^{q} y_{2}}{d t^{q}} \\
\frac{d^{q} y_{3}}{d t^{q}}
\end{array}\right]=\left[\begin{array}{c}
10\left(s(t)-y_{1}\right) \\
40 y_{1}-y_{1} y_{3} \\
4 y_{1}^{2}-2.5 y_{3}
\end{array}\right]+\beta\left[\begin{array}{c}
0 \\
\left(45 x_{1}-x_{1} x_{3}\right)-\left(45 y_{1}-y_{1} y_{3}\right) \\
4 x_{1}^{2}-4 y_{1}^{2}
\end{array}\right]
$$

where $s(t)=x_{2}(t)+m(t)$ is Channel transmission signal. $m(t)$ is the transmitted useful signal. We let $m(t)=\sin (t)$. Because $x_{2}(t)$ is the chaotic signal of broadband spectrum like noise, $s(t)$ is also broadband spectrum like noise. It is shown in Fig. 4(a). This is a chaotic security measure for secure communication. We can recover the transmitted useful signal after synchronization at the receiver terminal end. The recovered signal $M(t)$ is produced after passing through the subtractor as the difference between $y_{1}(t)$ and $x_{1}(t)$.It is shown in Fig. 4(b). Simulation results show that the system can restored the useful signals after about $0.5 \mathrm{~s}$ as showing in Fig.8.Hence, the secure communication scheme is effective.

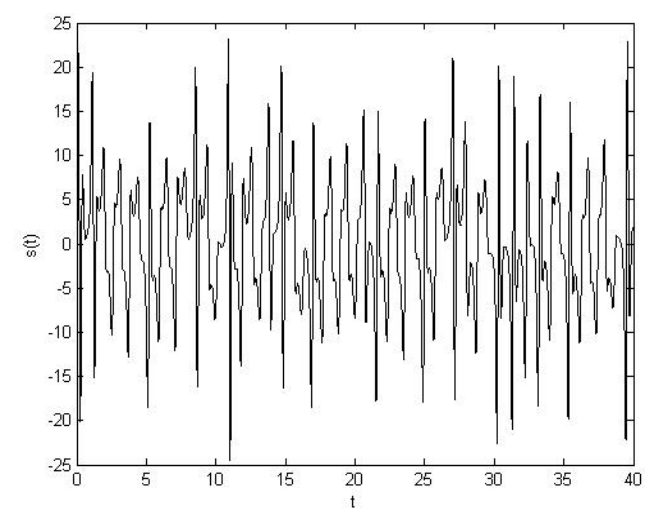

(a) 


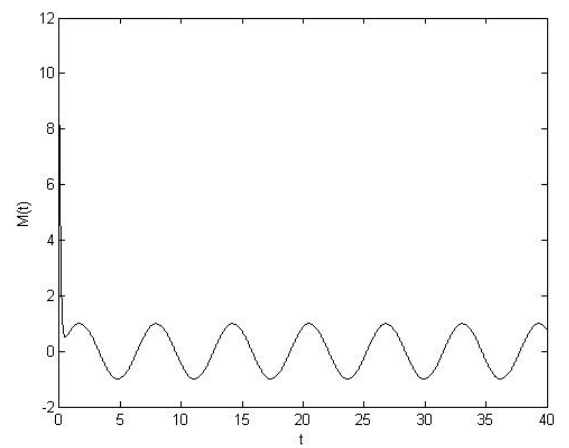

(b)

Fig. 4. Waveforms of transmitted signal $s(t)$ and recovered signal $M(t)$.(a) $s(t)$; (b) $M(t)$.

\section{Conclusion}

This paper developed the nonlinear-coupled method to make the fractional-order Liu systems synchronize. This method is simple and easy to achieve synchronization of fractional-order chaotic systems. Moreover, the linear matrix also influences synchronal time, and the systems with larger order will take less time up to synchronal state. Last, the method can be applied to secure communication.

\section{Acknowledgements}

This work was supported by the Natural Science Foundation of Fujian Province, China ( Grant No. 2012D127), and the Science and technology project of Fujian Provincial Department of Education ( Grant No. JA11264 ).

\section{References}

[1] Z. M. Ge, C. Y. Ou: Chaos, Solitons \& Fractals Vol. 35(2008), p. 705-717.

[2] X. Y. Wang, M. J. Wang: Chaos Vol. 17(2007), p. 033106-1-6.

[3] L. J. Sheu, H. K. Chen, J. H. Chen, L. M. Tam, W. C. Chen, K. T. Lin, Y. Kang: Chaos, Solitons \& Fractals Vol. 36(2008), p. 98-103.

[4] R. X. Zhang, S. P. Yang: Nonlinear Dynamics Vol. 69(2012), p.983-992.

[5] L. L. Huang, X. Qi: Acta Physica Sinica Vol. 62(2013), p.080507-1- 7.( In Chinese)

[6] H. J. Yu, N. Zheng: Acta Physica Sinica Vol. 57(2008), p.4712-4720. (In Chinese) 